Review paper

\title{
Recent advances in X-ray imaging of breast tissue: From two- to three-dimensional imaging
}

\author{
L. Heck *, J. Herzen \\ Biomedical Imaging Physics, Department of Physics and Munich School of BioEngineering, Technical University of Munich, James-Franck-Strasse 1, 85748 Garching, \\ Germany
}

\section{A R T I C L E I N F O}

\section{Keywords:}

Mammography

Breast Computed Tomography

Phase Contrast

Radiation Dose

Contrast Enhancement

\begin{abstract}
A B S T R A C T
Breast cancer is a globally widespread disease whose detection has already been significantly improved by the introduction of screening programs. Nevertheless, mammography suffers from low soft tissue contrast and the superposition of diagnostically relevant anatomical structures as well as from low values for sensitivity and specificity especially for dense breast tissue. In recent years, two techniques for X-ray breast imaging have been developed that bring advances for the early detection of breast cancer. Grating-based phase-contrast mammography is a new imaging technique that is able to provide three image modalities simultaneously (absorption-contrast, phase-contrast and dark-field signal). Thus, an enhanced detection and delineation of cancerous structures in the phase-contrast image and an improved visualization and characterization of microcalcifications in the dark-field image is possible. Furthermore, latest studies about this approach show that dosecompatible imaging with polychromatic X-ray sources is feasible. In order to additionally overcome the limitations of projection-based imaging, efforts were also made towards the development of breast computed tomography (BCT), which recently led to the first clinical installation of an absorption-based BCT system. Further research combining the benefits of both imaging technologies is currently in progress. This review article summarizes the latest advances in phase-contrast imaging for the female breast (projection-based and threedimensional view) with special focus on possible clinical implementations in the future.
\end{abstract}

\section{Background}

According to the World Health Organization (WHO), cancer is the second leading cause of death globally with 9.0 million deaths [1]. Thereby, breast cancer is the most common type of cancer worldwide and the leading cause of death among women under the age of 60 [2]. For 2018, it was expected that about $15 \%$ of women died from breast cancer among all cancer deaths [3]. For these reasons, the early detection of breast cancer is nowadays an unavoidable tool. The imaging techniques used so far in clinical environment are limited to mammography, ultrasound (US) and magnetic resonance imaging (MRI) where the former is used for screening. On the one hand, the introduction of these screening programs successfully demonstrated the decrease of the mortality rate by up to $45-58 \%$ in the USA [4] and $38-48 \%$ in Europe [5], but also worldwide [6-9]. On the other hand, values like the sensitivity (69-94\%) and the specificity (78-95\%) [10] as well as the positive predictive value (7-13\%) [11] greatly underline the potential to further improve the diagnostic image content of the female breast.
Particularly for woman with dense breast tissue, the sensitivity decreases further down to $30-48 \%[12,13]$. Due to the low soft tissue contrast in two-dimensional X-ray imaging, it is sometimes not possible to detect palpable masses or suspicious findings like cancerous lesions or cysts. Therefore, the overlap of diagnostically relevant tissue structures and thus the acquisition of clinically usable images is still a challenging topic in breast imaging.

Alternative non-radiographic-based techniques like US and MRI additionally support the diagnosis of breast cancer. Studies about MRI investigated that the sensitivity with $90 \%$ is very promising [14] while the specificity is comparably low [15]. However, MRI is a costly and time-consuming imaging procedure that requires an imaging expert while it suffers from a low resolution. A higher specificity for MRI can only be achieved by the injection of contrast agent. Thus, MRI is not ideal for people with contraindications like side effects of contrast agents, claustrophobia and if they have a pacemaker. Therefore, MRI is not suitable for breast cancer screening. US, however, is a technique from which mainly young females [16] or women with dense breast

\footnotetext{
* Corresponding author.

E-mail address: lisa.heck@tum.de (L. Heck).
} 
tissue benefit and which supports mammography results for a more reliable image evaluation. On the one hand, the sensitivity increases by the combination of these two techniques [14], but on the other hand, it leads to an increased number of false positive diagnoses and thus to overdiagnosis [17]. Since more than $20 \%$ of detected breast cancers are categorized as overdiagnosis, this remains a challenging topic in breast cancer detection [18].

As second-level examination, contrast-enhanced spectral mammography (CESM) based on dual-energy K-edge subtraction imaging is a technique which helps to overcome limitations of conventional mammography and is mainly used in the case of uncertain results. Findings that are not possible to detect in mammography become visible through the injection of iodinated contrast agent which accumulates in the newly formed blood vessels resulting from neo-angiogenesis of a tumor [19]. Disadvantages such as a higher applied mean glandular dose (MGD) and the use of contrast agents due to its toxicity [20] are also present, making CESM not an ideal imaging method to solve the problems of standard mammography. Consequently, the demand for an extension of projection-based X-ray imaging is growing over time: Digital breast tomosynthesis (DBT) is an already clinically used method which needs multiple projections of the compressed breast from different angles to obtain sliced images of the breast and thus limiting the effect of the superposition of breast tissue. Although studies have shown the advantage of DBT compared to standard mammography [21-23], recent research focusses more on the development of breast computed tomography to get an unlimited view of the female breast. Nevertheless, absorption-based X-ray imaging suffers from the inherently low soft tissue contrast, which makes tissue discrimination and diagnosis difficult. In 2014, an overview of the developments of several different phase-contrast imaging techniques applied to the field of breast imaging and their promising initial results concerning contrast enhancement was already presented [24]. In the recent years, further advances towards the clinical implementation of phase-contrast mammography and towards the extension to three-dimensional imaging have been achieved. In addition, the combination of phase-contrast imaging and computed tomography is greatly under research and seems to be very promising. The latest results are presented and summarized in this review article, but please note that due to the large number of publications in the field of breast imaging, only a small fraction can be presented in more detail in this manuscript.

\section{Two-Dimensional X-ray breast imaging}

\subsection{Phase-Contrast Mammography}

The demand for an imaging technique that provides more information about soft tissue contrast is very high due to the weak absorption contrast in biomedical samples. Nowadays, different phase-sensitive imaging techniques including propagation- and grating-based phasecontrast imaging are widely accessible for mono- and polychromatic Xray sources. These methods benefit from an improved visualization of soft tissue since the phase shift of the X-ray wave front can be measured. Over the last two decades, research on propagation-based phasecontrast mammography at synchrotron facilities like at the SYRMEP (synchrotron radiation for medical physics) beamline of the Elettra synchrotron in Trieste (Italy) has been strongly promoted, starting with a proof-of-principle and successfully ending up with a clinical study including more than 70 patients in total [25-29]. While imaging with synchrotron radiation benefits from an optimized contrast-dose ratio by eliminating X-ray photons that contribute to dose rather than contrast, there are also limitations of large synchrotron facilities such as limited availability, high costs and distance from hospitals. In first proof-ofprinciple studies, mammography and breast tomosynthesis were performed at a compact synchrotron source using the grating-based approach to overcome the aforementioned disadvantages while benefiting from the lower dose compared to clinical measurements
$[30,31]$. With the grating-based phase-contrast imaging approach, it is additionally possible to obtain a dark-field signal that relates to smallangle scattering of structures such as microcalcifications in the breast $[32,33]$. The improvement of the diagnostic image content of the latter technique seems to be very promising as it can be conducted with clinically available polychromatic X-ray sources. In the following, the latest developments including different fields of applications for gratingbased phase-contrast mammography and their final implementation towards clinical imaging are summarized.

\subsection{Grating-Based Mammography}

\subsubsection{Classification of Microcalcifications using X-ray Dark-Field Imaging}

Since microcalcifications in the breast are visible in more than half of non-palpable malignant tumors [34] and since they are the main reason for the detection of ductal carcinomas in situ (in 85-95\% of cases) [35] as well as for invasive cancers [36], their diagnostics and early detection is very important for the diagnosis of breast cancer. Wang et al. initially presented the classification of microcalcifications using the non-invasive combination of absorption-based and dark-field mammography imaging [37]. Based on phantom and fresh sample measurements, they successfully presented the distinguishability of microcalcifications of type I and II while the dose exceeds the clinical limit. In the following years, however, these results were controversially discussed in the literature [38-40]. In 2016, Scherer et al. further examined the benefit of the darkfield signal by the investigation of the micromorphology and showed that the diagnostic content is enhanced so that invasive procedures such as biopsies could be reduced [41]. In their results, they initially presented that the mean $\mu / \mathcal{E}$-ratio, which corresponds to a ratio between the absorption power $\mu$ and the scattering power $\varepsilon$, is not depending on the orientation of the microcalcification cluster or whether they are embedded in any material. Based on that, 15 biopsies of microcalcification clusters were measured in a micro-CT investigating the $\mu / \mathcal{E}$ ratio and its dependence on the inner structures of the clusters. Thereby one differentiates between the following groups of microcalcifications: ultra-fine, fine, pleomorphic and coarse. A scatter plot of the $\mu / \mathcal{E}$-ratio is visible in Fig. 1(A) clearly showing that the slope and the pattern varies between the different classes. The blue marked, ultra-fine und uniform microcalcifications with a radius of $75 \mu \mathrm{m}$ have a high scattering and only a low absorption power resulting in a flat slope of the data points. Furthermore, the low variance underlines the small spread of the data which establishes a uniform and regular particle size within these clusters. Fine structured microcalcifications clusters (red labelled) with larger particle sizes of $175 \mu \mathrm{m}$ have a higher $\mu / \mathcal{E}$-ratio and a larger variance caused by a higher absorption and lower scattering power than before and caused by some larger particles. However, the highest variance of 0.89 can be found for microcalcifications with a pleomorphic microstructure. These clusters consist of a mix of small and larger calcium particles leading to a steeper slope than for ultra-fine and fine calcifications. The green framed microcalcifications with a coarse microstructure and a size of about $700 \mu \mathrm{m}$ have the steepest slope in the scatter plot and therefore absorb more than they scatter. Summarizing, the $\mu / \mathcal{E}$-ratio increases from ultra-fine to coarse microcalcification clusters and thus, it is possible to determine the micromorphology of microcalcifications by characterizing the slope and the spread of the scatter plots. In a second step, the focus was on the investigation of a possible discrimination between benign and malignant microcalcification clusters. For this purpose, 31 microcalcification clusters are investigated where twenty belong to benign and eleven to malignant findings (ductal carcinoma in-situ). The corresponding scatter plot and histogram of this study is presented in Fig. 1 (B-C). These two plots show that benign microcalcification clusters consist mainly of ultrafine or fine microstructures, while malignant microcalcifications rather dominate the area of pleomorphic and coarse microtextures. This observation is underlined by the fact that $68 \%$ and $69 \%$ of polymorphic and coarse microstructures belong to the malignant microcalcifications and only 


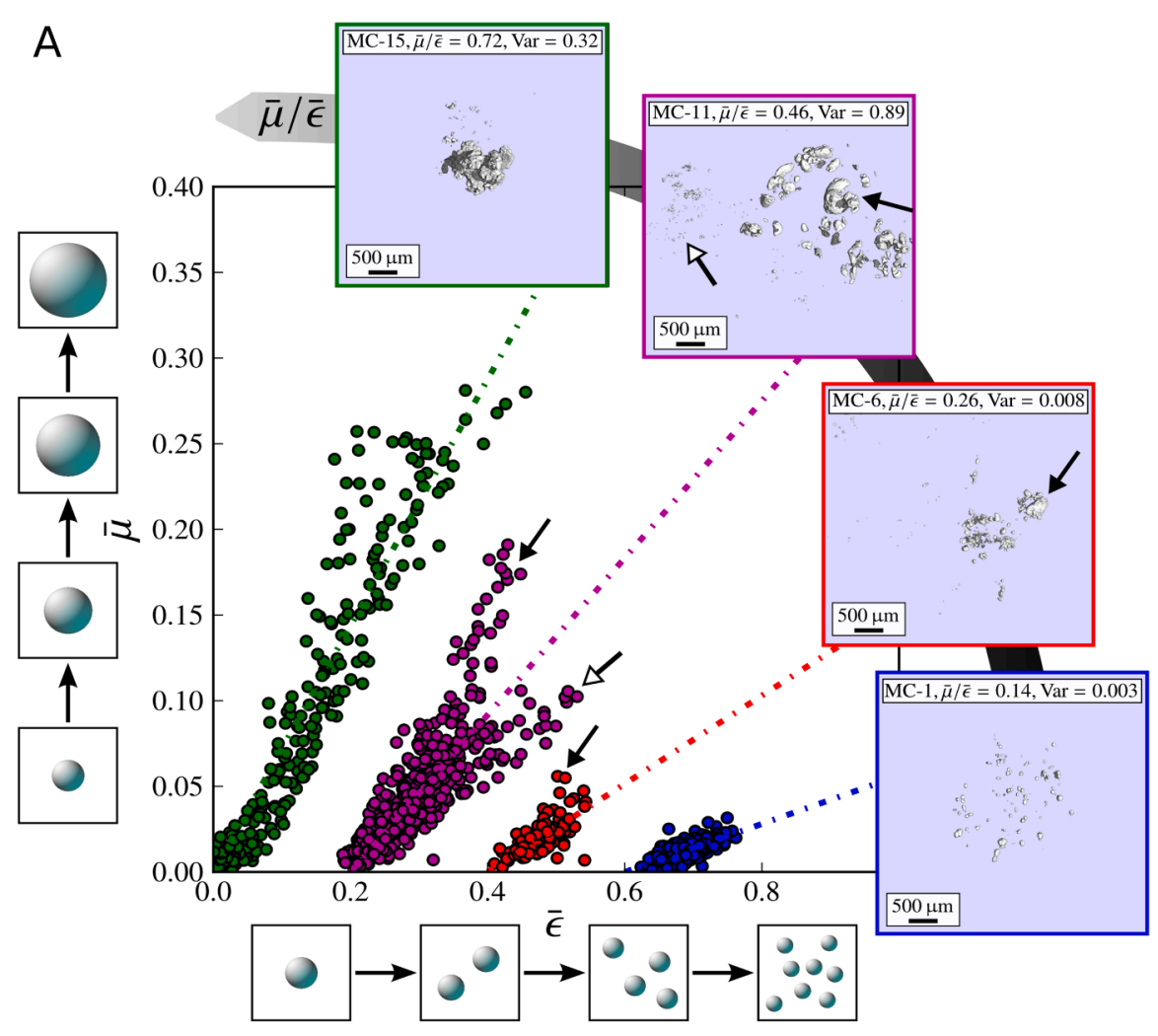

Fig. 1. (A) Visualization of the absorption $\mu$ and scattering power $\varepsilon$ dependency of microcalcification clusters on their inherent micromorphology comparing coarse (green), pleomorphic (purple), fine (red) and ultrafine (blue) microtexture classes [41]. The calculated mean $\mu / \varepsilon$-ratios differs strongly for the four different classes. Arrows indicate the distinct diversification in grain size of the respective micromorphology class which is consistent with the plotted data. (B) Comparison the absorption $\mu$ to scattering power $\varepsilon$ of 20 microcalcifications histopathologically associated with a benign finding (blue) and 11 microcalcifications associated with a ductal carcinoma in-situ (red). The distribution of the $\mu / \varepsilon$-ratios for benign and malignant microcalcifications obtained from (B) are compared in a histogram (C). Microcalcifications with benign findings result in $\mu / \varepsilon$-ratios corresponding to ultra-fine and fine microtextures, while malignant microcalcifications predominate within ratios intended for pleomorphic and coarse microstructures. Images reprinted from Scherer et al. with permission from the authors [41]. (For interpretation of the references to colour in this figure legend, the reader is referred to the web version of this article.)
B

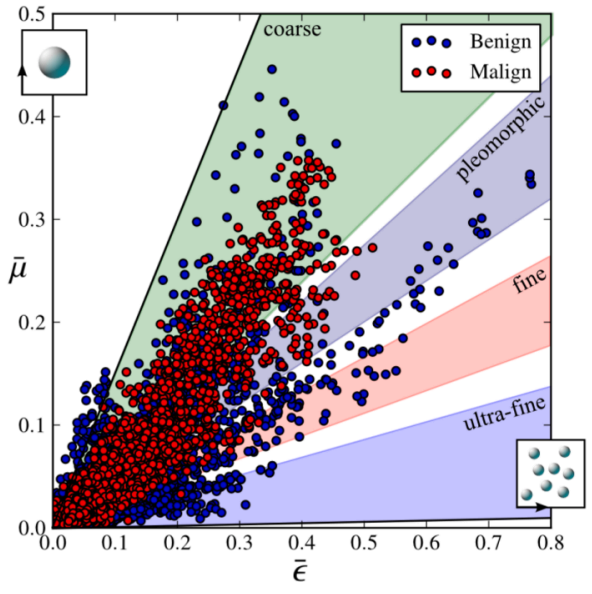

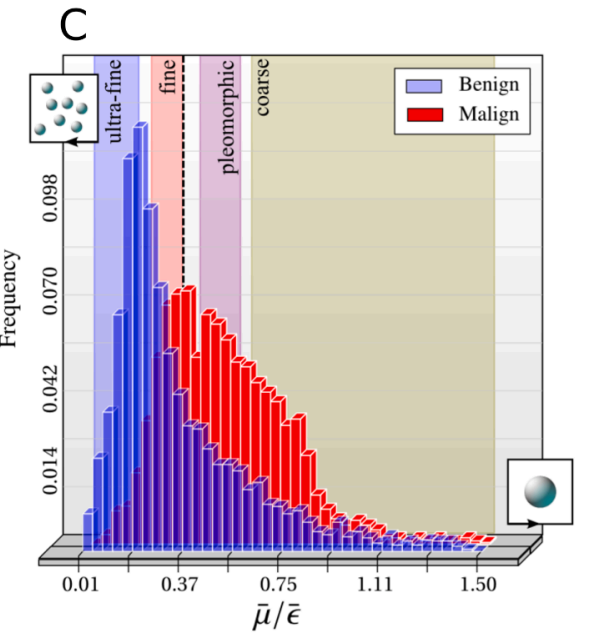

$12 \%$ and $37 \%$ of calcifications are associated with ultrafine or fine microstructures. Although this proof-of-principle study suffers from a MGD exceeding clinical dose levels, Scherer et al. propose this method as a possible in-situ application instead of invasive procedures such as biopsies. A further study in the last year with a larger patient group (62 samples) deals again with the question whether one is able to distinguish between benign and malignant microcalcifications [42]. According to a statistical analysis, the differentiation is not of statistically significant relevance. Nevertheless, the authors are in accordance with Scherer et al. that this non-invasive technique has the potential for a better visibility of different types of microcalcifications and simultaneously a reduction of the false positive biopsy rate.

\subsubsection{Improvements by Bi-Directional Phase-Contrast Imaging}

Previous studies have already proved the benefits of the differential phase contrast for the image diagnostics in projection-based breast imaging. Nevertheless, these results suffer from different disadvantages, e.g. the anisotropic imaging sensitivity of the differential phase-contrast signal due to the gratings only being oriented in one direction. Thus, the electron density is only accessible perpendicular to the grating lines. Added to this is the possibly undetected dark-field signal, if it is emitted parallel to the gratings [43]. Consequently, the risk of diagnosing more false-positive results remains if oriented structures are overlooked. In order to overcome these problems, Scherer et al. investigated twodirectional phase-contrast mammography based on an invasive ductal carcinoma [44]. In this study, two scans are recorded that are orthogonal to each other. The results of the bi-directional scan for the differential phase-contrast, the dark-field signal and the fused images are delineated in Fig. 2. Thereby, the arrows in the figure legend indicate the scanning direction during the phase stepping procedure which was performed in $\mathrm{x}$ - and $\mathrm{y}$-direction. On the one hand, it is clearly visible that the vertically oriented tumor branches which are marked by the red box 

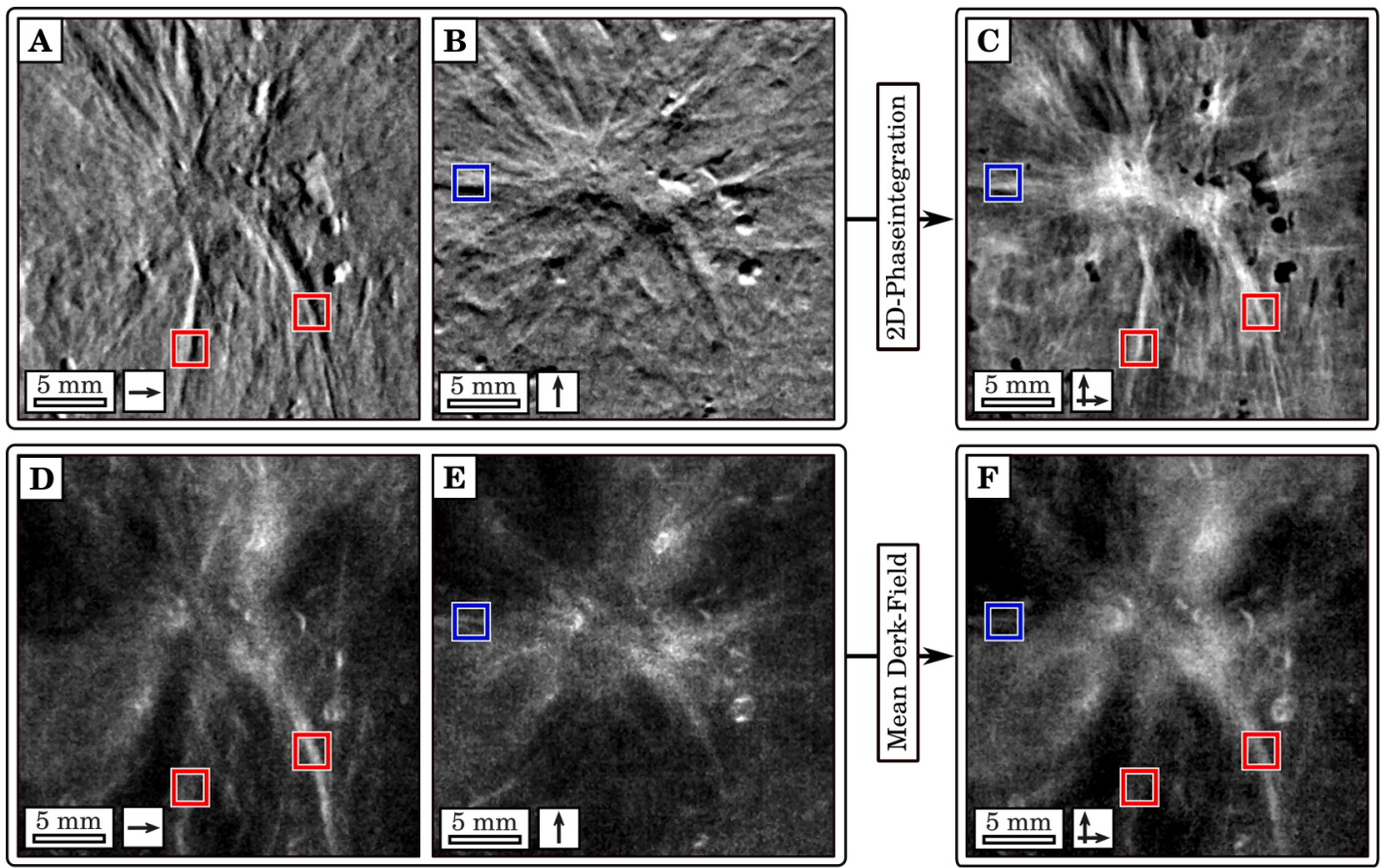

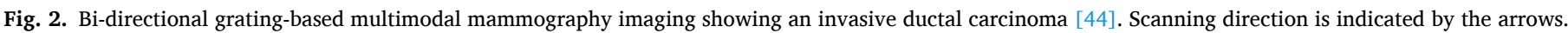

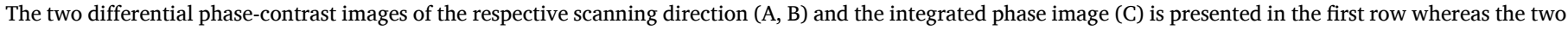

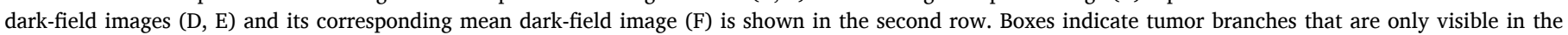
images acquired by scanning in $\mathrm{x}$ - and $\mathrm{y}$-direction. Images reprinted from Scherer et al. with permission from the authors [44].

in subfigures (A) and (D) are not detectable in the orthogonally oriented images (B) and (E). On the other hand, also the horizontally detected tumor branches, highlighted by the blue boxes, are not visible in the images oriented in the $\mathrm{x}$-direction. This is valid for both the differential phase-contrast and for the dark-field signal. Thus, this study confirms the directional dependence of the scanning direction. Only the twodimensional phase integration (cf. Fig. 2(C)) and the mean dark-field signal (cf. Fig. 2(F)) where the information of both scans was fused to one image reveals the full diagnostic image content of the tumor branches orientated in different directions. In addition to that, the authors compared the two-dimensional integrated phase-contrast to the conventional dose-compatible absorption-contrast image. This comparison again underlines the benefit of this imaging approach since more fine details are visible (three tumor lesions vs. one pervasive tumor structure) and consequently the soft-tissue contrast is significantly improved. Thus, this study proves the benefit of multi-directional imaging in order to fully evaluate the extent of a cancerous lesion independently from its orientation.

\subsubsection{Dose-Compatible Phase-Contrast Mammography at a Compact Synchrotron Source}

The further development of inverse Compton sources, which benefit from their compactness in contrast to large-scale synchrotron facilities, opened up the possibility of performing mammography studies with dose-optimized X-ray spectra in recent decades. With this approach, the applied MGD can be further reduced compared to studies conducted with polychromatic X-ray sources since low-energy X-ray photons which only contribute to the dose but not to the contrast are successfully eliminated by the quasi-monochromatic X-ray spectrum. In a first mammography study at the Munich Compact Light Source, Eggl et. al investigated four samples focusing on different aspects [31]. First, the authors showed an improved detection of cancerous lesions in the
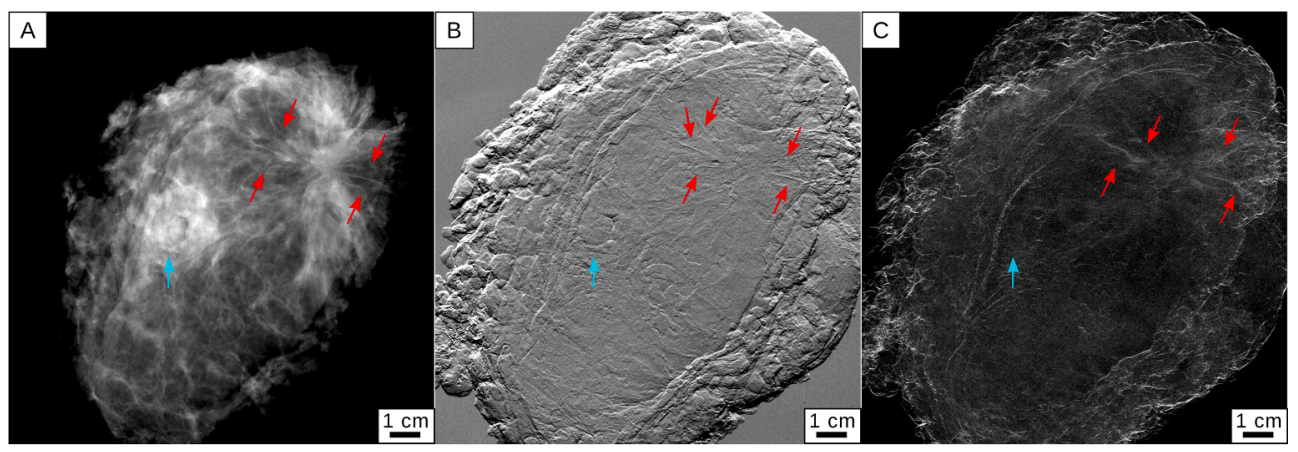

Fig. 3. Grating-based multimodal mammography imaged at the Munich Compact Light Source showing the absorption-contrast (A), differential phasecontrast (B) and dark-field image (C). Images are taken at an X-ray energy of $25 \mathrm{keV}$ and a MGD of $0.8 \mathrm{mGy}$ which is comparable to the clinically applied dose in this study. The tumor lesions are indicated by red arrows whereas the mamilla is indicated by a light blue arrow. Fine tumor branches which originate from the tumor and perfusing the surrounding tissue are better visible in the differential phase-contrast image (B) and also in the dark-field image [31]. Images reprinted from Eggl et al. with permission from the authors [31]. (For interpretation of the references to colour in this figure legend, the reader is referred to the web version of this article.) 
differential phase-contrast image which is exemplary depicted in Fig. 3. Fine tumor branches which originate from the tumor while perfusing the adjacent tissue are better detectable in the differential phase-contrast image than in the absorption-contrast image. In the dark-field image, these branches are also visible, but only to a reduced extent. Although images are taken with gratings in the beam path, the applied MGD is slightly lower than in clinical in and ex vivo mammography. Secondly, Eggl et al. investigated the detection of microcalcifications at a significantly reduced MGD ( $0.3 \mathrm{mGy}$ instead of $1.4 \mathrm{mGy}$ ). In a dose study performed with a mammographic accreditation phantom, an analysis of the contrast-to-noise ratio (CNR) was finally conducted to quantitatively compare experimentally and clinically recorded images. It has been shown that the CNR of absorption-only images taken with the monochromatic radiation exceed clinical imaging at the same MGD. In addition, the CNR slightly outperforms clinical imaging while MGD is reduced by $20 \%$. For the grating-based multimodal imaging approach, one additionally benefits from a better depiction of the fiber structures as already initially mentioned. An additional resolution analysis shows that the monochromatically taken images achieve a higher resolution than the clinical images. All in all, this study shows that one benefits on the one hand from the dose reduction which is an important issue in $\mathrm{X}$ ray breast imaging and on the other hand from the gain in information through the grating-based imaging approach. Medical imaging at compact inverse Compton sources is currently not feasible in the clinical environment, but more likely to be installed there in the far future since the financial and spatial requirements are low compared to largesynchrotron facilities which also provide dose-optimized X-ray spectra.

\subsubsection{Enhanced Delineation of Breast Cancer Features}

Since the last review paper, where it has already been presented that a better visualization of breast lesions have been achieved [24], even more studies were published that have investigated the improved detection of tumor lesions by grating-based phase-contrast imaging $[45,46]$. Thereby, one publication by Grandl et al. mainly focusses on the enhanced delineation of breast cancer features in multifocal carcinomas [45]. Therefore, the phase-contrast and the dark-field images are evaluated in an ex vivo study with three freshly dissected mastectomy samples. The results are finally compared to the clinical standard imaging procedures like ultrasound, histopathology, MRI and absorptioncontrast mammography. Samples are selected to include one bifocal carcinoma and one tri- and one multifocal invasive carcinoma. The three samples reveal different results: The first result shows that by considering the dark-field signal for medical diagnosis, it is possible to detect more tumor nodules than with clinically conventionally used imaging methods. While in conventional mammography and dynamic MRI only two tumor lesions were detected, a third one becomes visible in the darkfield image. Due to the low spatial resolution of MRI, it only appears like one enlarged tumor lesion instead of two smaller nodules. Finally, histopathology proved the third tumor lesion to be a calcified tumor nodule which is the reason why it is better visible in the dark-field instead of the phase-contrast image. Line plots through the three tumor lesion as well as the calculation of the contrast-to-noise ratio proved the enhanced detection of the tumors in the dark-field compared to the absorptioncontrast image. In addition to that, the dark-field image of the second sample also improved the depiction of tumorous strands. Although these strands are visible in all conducted image modalities, the dark-field image obtains the best contrast and additionally more information about the extension of the strands while perfusing the surrounding tissue. With this further information, more preoperative diagnostic would have been necessary in order to fully investigate the extent of the tumor. The third sample contains two tumors in total which were successfully detectable in all clinical and experimental image modalities. But by taking the phase-contrast and dark-field information additionally into account, it is possible to clearly see the margins of the tumor and thus enhance the diagnostic image content. For this study, it must be considered that all measurements have not been performed at a clinically compatible dose level, but two of the three samples were measured in the bi-directional imaging mode. In order to further investigate the benefit of grating-based phase-contrast mammography, studies with larger patient cohorts must be conducted as done by Hauser et al. with totally 33 samples including different types and grades of breast cancer [46]. In their study, they focused on the comparison between clinical absorption-contrast imaging to differential phase-contrast mammography in a reader study including six radiologists. Their results underline the conclusion presented so far: Superior image quality due to a sharper delineation of cancerous lesions, microcalcifications and anatomical structures. Consequently, they concluded a superior image quality assessment by the complementary information obtained by this novel imaging approach [46].

\subsection{Grating-Based Mammography towards Clinical Implementation}

One of the main limitations of grating-based phase-contrast mammography with polychromatic X-ray sources remains the applied MGD which in the previously presented cases exceeds the officially stated limit of $2.5 \mathrm{mGy}$ per view [47]. This dose problem should be fundamentally solved if grating-based phase-contrast mammography is aimed to be a prospective application in daily clinical use. Hence, recent studies successfully demonstrated more dose-compatible results for this technique [48-50]. For an experimental laboratory setup, Scherer et al. achieved to address the mainly discussed points of long acquisition times and high MGD for a mammographic phantom as well as for a freshly dissected mastectomy specimen. The previously used setup was optimized with the following steps: Increasing the source-to-sample distance and decreasing the number of phase steps, changing the detector to a more efficient and faster one, thus decreasing the exposure time per phase step down to one second and reducing the substrate thickness of the analyzer grating. In addition, a tissue equivalent absorber (PMMA) was placed in the beam path for the acquisition of flat field images in order to prevent detector saturation, shadowing artifacts (since comparable spectra for both measurements are obtained), variations in the fringe visibility, an artificial dark-field signal and to provide a quantitative image signal. Thus, a MGD of below $2.5 \mathrm{mGy}$ is achieved for both the phantom ( $2.07 \mathrm{mGy}$ ) and the fresh specimen ( $2.2 \mathrm{mGy}$ ). However, some points remain that limit potential clinical use, such as a restricted field-of-view (FOV) due to the size of the detector and the gratings. The latter problem is currently also under research focusing on the production of large FOV gratings [51,52]. Nevertheless, Köhler et al. finally succeeded in building a grating-based phase-contrast full-field mammography imaging device that meets the main clinical requirement of scanning the entire FOV [49]. In their approach, they implemented a Talbot-Lau interferometer into an existing clinical imaging device (Philips MicroDose L30 FFDM) and integrated a slit-scanning system. Thus, they achieved a FOV of $16 \times 19.6 \mathrm{~cm}$, a scanning time between 10 and $15 \mathrm{~s}$ and a MGD of approximately $1.9 \mathrm{mGy}$. Although this study lacks fresh breast samples, low phase sensitivity and some artifacts in the processed images, this result is a fundamental milestone for mammography finally bringing phase contrast into the clinical environment after years of research. In recent research, the development of this clinical imaging device for mammographic applications has been continued. Therefore, the optimal parameters were investigated for the design of a grating-based mammography system which reveals that the highest sensitivity is achieved the smaller the grating pitches are and that it additionally depends on which signal type is optimized [53]. Then, by optimizing on the differential phase-contrast signal, this knowledge is used to build up the first clinical full-field grating-based mammography system (again a modification of a Philips MicroDose system) which is compatible with the clinical requirements [54]. In a study with five mastectomy specimens, Arboleda et al. successfully demonstrated the clinical feasibility of such an imaging system. It additionally includes fused attenuation- and phase-contrast images whose benefit has still to be evaluated. One exemplary image of this 
study is shown in Fig. 4. Although the MGD is still higher (1.6 mGy and $2.0 \mathrm{mGy}$ ) than in other clinical mammography systems, this can be justified by the additional information obtained with grating-based phase-contrast imaging, which provides more diagnostic value.

\section{Three-Dimensional X-ray Breast Imaging}

\subsection{Overview of Absorption-Based Breast Computed Tomography (BCT)}

The first efforts towards absorption-based breast computed tomography (BCT) were already made in the 1970's by Chang et al. [55-57]. Just like the nowadays available dedicated BCT's, the patient lies in prone during the measurement. At that time, the breast has additionally been immersed in a water bath with body temperature water [56,57]. The CT scan was performed with long scan times $(10 \mathrm{~s})$ for a $1 \mathrm{~cm}$ thick slice and a large pixel size $(1.56 \mathrm{~cm})$ [57]. Although the studies have shown improvements for breast cancer detection especially by the combination with a contrast medium enhancement technique (pre- and postinjection images) injecting diatrizoate meglumine [56], technical improvements concerning, for example, the pixel size for the detection of lesions that are smaller than $1.55 \mathrm{~mm}$ or microcancer smaller than 1 $\mathrm{mm}$ as well as the exposure times were necessary. Through the developments in various technical areas since the last century, the efforts in developing a BCT have been resumed in the last two decades. In 2002, Boone et al. evaluated the potential of dedicated BCT and concluded that BCT should be further investigated due to its high potential for breast cancer diagnosis, as the simulated average MGD achieved for an $80 \mathrm{kVp}$ measurement was comparable to two-view mammography [58]. A few years later, initial experimental results of dedicated BCT systems using an indirect conversion detector (cesium iodide scintillator) with a tube voltage of $80 \mathrm{kVp}$ were published underlining the previously evaluated potential of BCT for the improvement of detecting and characterizing lesions in different ways [59-61]. In order to reduce motion artifacts, the scan time of the first prototype scanner with about $17 \mathrm{~s}$ [59-61] was reduced down to $9 \mathrm{~s}$ for a second scanner [59]. The applied MGD differs in the various studies: Depending on the breast size, the MGD was between 4 and $16 \mathrm{mGy}$ [61], between 2.5 and $10.3 \mathrm{mGy}$ [60] or comparable to two-view mammography images [59]. It has been shown that the complexity of the depiction of the three-dimensional breast volume
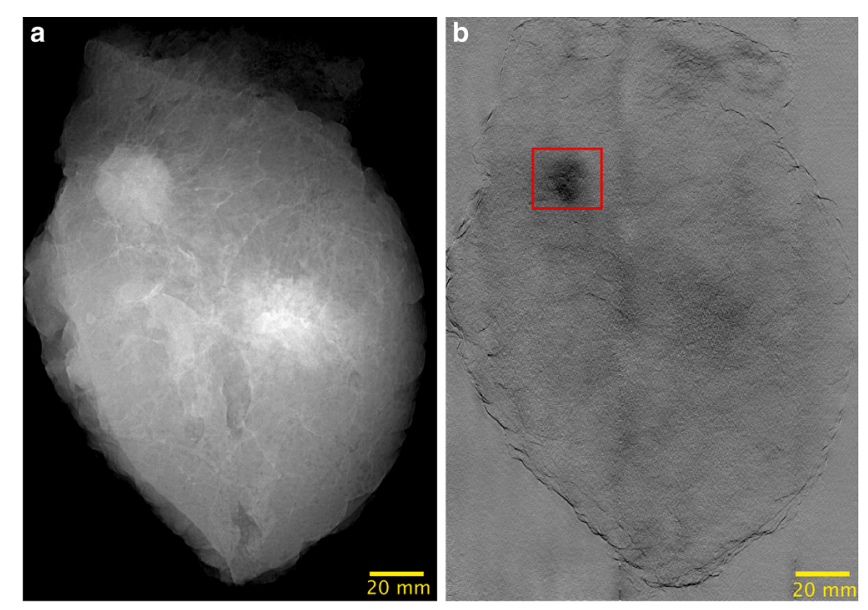

Fig. 4. Example for grating-based phase-contrast mammography implemented in a clinical imaging device (Philips MicroDose system) [54]. An image fusing the attenuation and the differential phase-contrast image is shown in (a) and the corresponding dark-field signal which provides a significantly improved detection of the cancerous area which is indicated by the red square. The applied MGD for this sample is $2.0 \mathrm{mGy}$ and thus in a clinically allowed dose range. Images reprinted from Arboleda et al. with permission from the authors [54]. (For interpretation of the references to colour in this figure legend, the reader is referred to the web version of this article.) helps imaging of anatomical structures like microcalcifications, breast lesions and tumor masses $[59,60]$. Only the benefit regarding the distinction between benign and malignant lesions could not be proven [60]. In addition to that, all these studies showed an enhanced visualization of tumors for contrast enhanced BCT by the injection of iodinated contrast agent [59-61]. Consequently, the detection rate of malignant breast lesions but also the applied MGD (8-32 mGy) increased [61]. One of the major discussed issues remaining is the applied radiation dose which should be as low as reasonably achievable (ALARA principle). The introduction of the photon-counting detector technology with several benefits compared to conventionally used flat panel detectors [62] with high quantum efficiencies helps to further decrease the MGD in BCT [63-66]. In 2011, Kalender et al. introduced a high resolution spiral BCT with a very low MGD (below $5 \mathrm{mGy}$ ) [63] and short acquisition times (below ten seconds) $[63,64]$. In contrast to the initially mentioned BCT developments, these measurements have been performed with a lower tube voltage of $60 \mathrm{kVp}$ and an additional $3 \mathrm{~mm}$ Aluminum filtration $[63,64]$. The used cadmium telluride-based photon-counting detector with a pixel size of $100 \mu \mathrm{m}$ aims to achieve an absorption efficiency of more than $99 \%$ which is very beneficial taking the radiation sensitive breast tissue in account. In their initial results, Kalender et al. evaluated the feasibility of a spiral BCT with a cylindrical breast phantom with varying diameter from 8 to $18 \mathrm{~cm}$ [63]. Simulations as well as experimental measurements have shown that microcalcifications and soft tissue lesions are detectable by only applying a MGD of 3 mGy [63]. Later, Kalender et al. published results of a study containing ten surgical lumpectomy specimens and one larger mastectomy specimen. In this study, BCT is compared to standard mammography and DBT in order to evaluate the benefit of BCT [64]. The results once again underline the superior detection of several different structural details of the breast, while surpassing clinically conventional imaging techniques such as mammography and DBT [64]. The foundation of different companies strongly working on bringing BCT into the clinics emphasizes the great potential of this imaging technique. Thereby, the best example is the german company $A B-C T$ - Advanced Breast-CT which developed a clinical device called nu:view [65] that is installed - among others - at the University Hospital of Zurich. The first results of clinical in vivo BCT imaging have just recently been published by Berger et al. showing high image quality, low radiation dose (between 4.4 and $5.7 \mathrm{mGy}$ obtained by Monte-Carlo simulations) and improved patient comfort [66]. In Fig. 5, an example of the BCT with (right column) and without (left column) the injection of contrast media is shown. A retroareolar lesion is visible in all the three views, but better detectable after the contrast medium administration. An additional lesion can finally be recognized in the sagittal view. Please note that due to the scope of this review, the publications mentioned in this subsection do not include all studies and efforts performed so far for absorption-contrast BCT. A full overview of all dedicated BCT systems developed at different universities is already given by Sarno et al. [67].

\subsection{Phase-Contrast Breast Computed Tomography}

In order to fully exploit the previously presented advantages of phase-contrast imaging and computed tomography for the female breast, research additionally focusses on the combination of both techniques.

\subsubsection{Initial Results for Grating-Based Phase-Contrast Breast Computed Tomography (phase-contrast BCT)}

In the previous review article, initial results for phase-contrast BCT, which are mainly limited to measurements at synchrotron facilities, have already been summarized [24]. Since then, further studies have been conducted on this topic, focusing for example on breast lesions such as fibroadenomas [68] or intraductal carcinoma in situ (DCIS) [69] or the quantitative characterization of breast tissue [70] using the grating-based approach with conventional X-ray sources. In a first study 


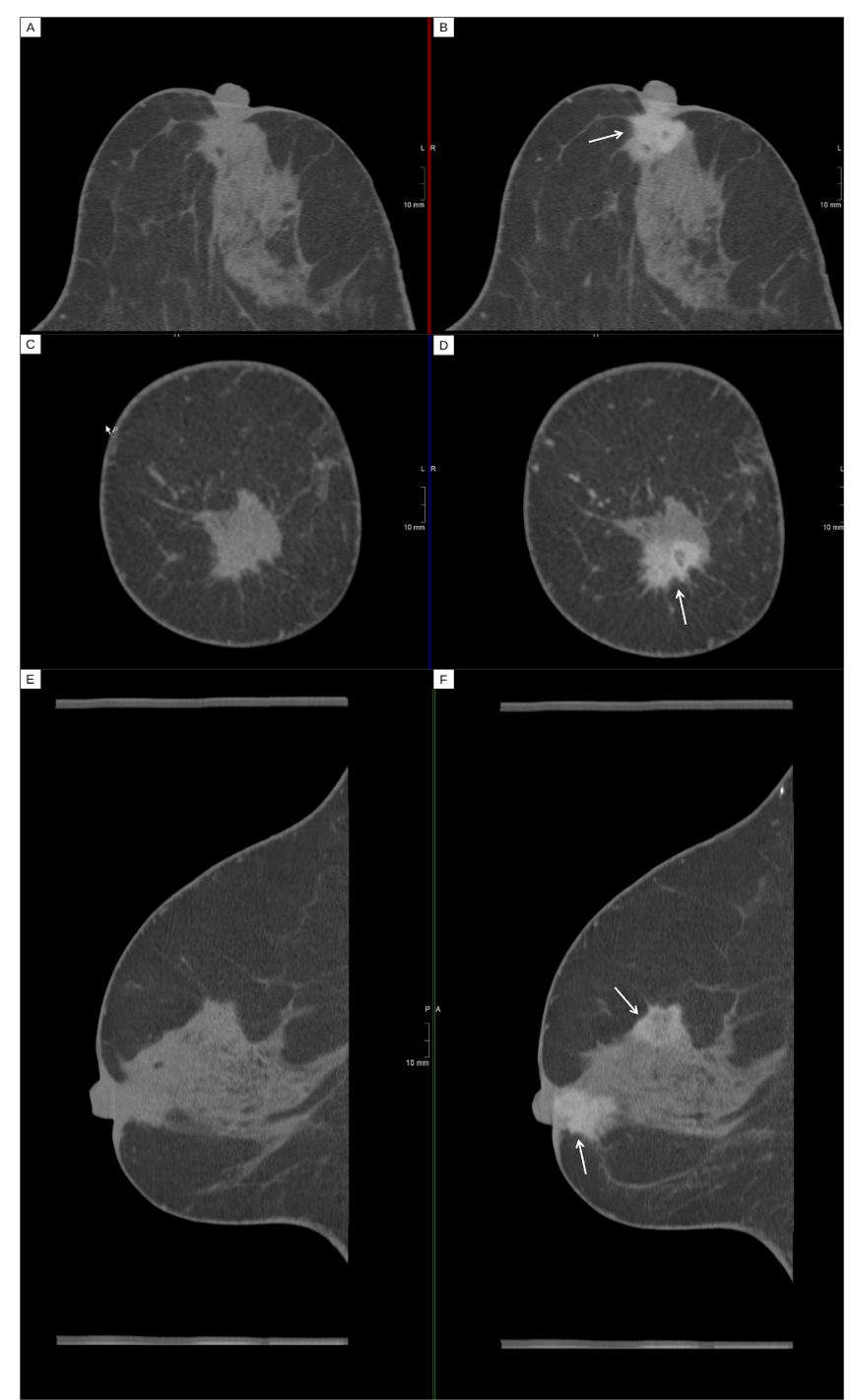

Fig. 5. Example of in vivo absorption-contrast breast computed tomography including the axial (A, B), the coronal (C, D) and the sagittal (E, F) view. The images in the left column are recorded without and the images in the right column with contrast medium. Patient with nipple retraction and multicentric invasive ductal carcinoma. The patient was diagnosed with a retroareolar lesion (invasive ductal carcinoma) which is better visible after contrast medium administration. The same lesion is visible in the three views. In the sagittal view an additional lesion can be recognized in the upper quadrants of the breast. Images were provided by Magda Marcon.

with a total of nine samples, a benign breast lesion, the so-called fibroadenoma, was investigated [68]. Its characterization is still a challenge with the current state of the art since the differentiation with a potentially malignant phyllodes tumor remains difficult. In this case, one could benefit from phase-contrast BCT in order to further specify the diagnosis of the lesion regarding malignancy or benignity. In this study, the CT slices were matched to histological results for a better comparison. One image is exemplary shown in Fig. 6 including the comparison between histology, absorption- and phase-contrast BCT. While the absorption-contrast BCT does not reveal any information about the internal breast tissue structure, the phase-contrast BCT clearly depicts the bright strands of the fibrous tissue and the small adipose tissue spots. An exemplary sample image for this study is shown in Fig. 6 (D-F) underlining the observations described above. In the publication of Grandl et al., these results are also confirmed by a histogram analysis, where it is not possible to distinguish between water, formalin and the fibroadenoma in the absorption (only one common peak), but in the phase- contrast image (three individual peaks). Concluding and comparing absorption-contrast to phase-contrast imaging, all samples within this study emphasize the fundamental improvement of the visualization and the resulting discrimination of finer structures within the sample. In addition, fiber strands, the borders of tumors and other breast lesion features are better visualized so that a more reliable characterization appears to be possible for the diagnosis of fibroadenomas. Please note that this study was conducted at a dose well above the clinically acceptable MGD and not with complete mastectomy specimens. Thus, this technique might currently be more useful as a non-invasive technique which serves as an additional support during the analysis from histology. Consequently, the combination of both techniques can lead to a reduction of false positive findings, the recall rate and biopsies. With this technology, Willner et al. quantitatively characterized breast tissue at the European Synchrotron Radiation Facility and with a polychromatic X-ray source [70]. In their study with samples including a benign phylloides tumor, an invasive lobular carcinoma and a fibroadenoma, they successfully estimated the corresponding phase-contrast Hounsfield units (HUp) from the given electron density or elemental composition and compared them to present literature. Values calculated at the synchrotron are finally confirmed by the results conducted with a laboratory setup. Most of the calculated HUp successfully agree with the literature, but there are also some discrepancies to the literature values present coming from the higher spatial resolution during this study. Since the values for different tumor types are all around 45 and 50 HUp and thus its differentiation remains challenging, a study containing more samples is necessary to further clarify the differences between several tumor types. Nevertheless, this study successfully demonstrated the feasibility of quantitative breast tissue characterization using grating-based phase-contrast computed tomography at a polychromatic $\mathrm{X}$-ray source, the results of which are of special interest for the design of suitable phantoms and the performance of simulations. In another study with a polychromatic X-ray source, Hellerhoff et al. examined the benefit of this technique for the detection and assessment of DCIS including totally four samples [69]. DCIS concerns that one fifth of all detected breast cancers and in most of the cases its extent and hence its sensitivity is comparatively low and underestimated. In this study, the comparison between absorption-based, phase-contrast BCT and histology was drawn again. On the one hand, for the absorption-contrast images, the obtained results have a very low contrast (e.g. for vessel walls) and a discrimination of ductal and glandular structures is not feasible. Nevertheless, the detection of microcalcifications is clearly visible. On the other hand, the phase-contrast imaging modality clearly reveals the shape of an invasive tumor including the surrounding DCIS, dilated ducts and the improved depiction of vessel walls. One example of this study is presented in Fig. 6 (A-C) where the dilated ducts are more clearly visible in the phase-contrast than in the absorption-contrast image. The results obtained so far are finally confirmed by the corresponding histological section slices. In conclusion, absorption-contrast images were outperformed by the correlation of phase-contrast BCT and the histopathological analysis. But although one benefits from the three-dimensional view of BCT, which helps to support histological evaluation in order to identify suspicious areas, one must consider the major limitation that the spatial resolution of phase-contrast BCT cannot compete with histological analysis.

\subsubsection{Propagation-Based Phase-Contrast Imaging at the Elettra Synchrotron Trieste and the Australian Synchrotron}

Another technique that has evolved in recent years is propagationbased rather than grating-based phase-contrast imaging for computed tomography of the female breast, as this approach benefits from the fact that there are no gratings in the beam path that negatively affects the MGD. Several advances were achieved and different aspects investigated in the latest time which have been performed at the SYRMEP beamline at the Elettra Synchrotron in Trieste [71-79]. After an initial feasibility study [79], first studies published in 2018 present the first full-volume 

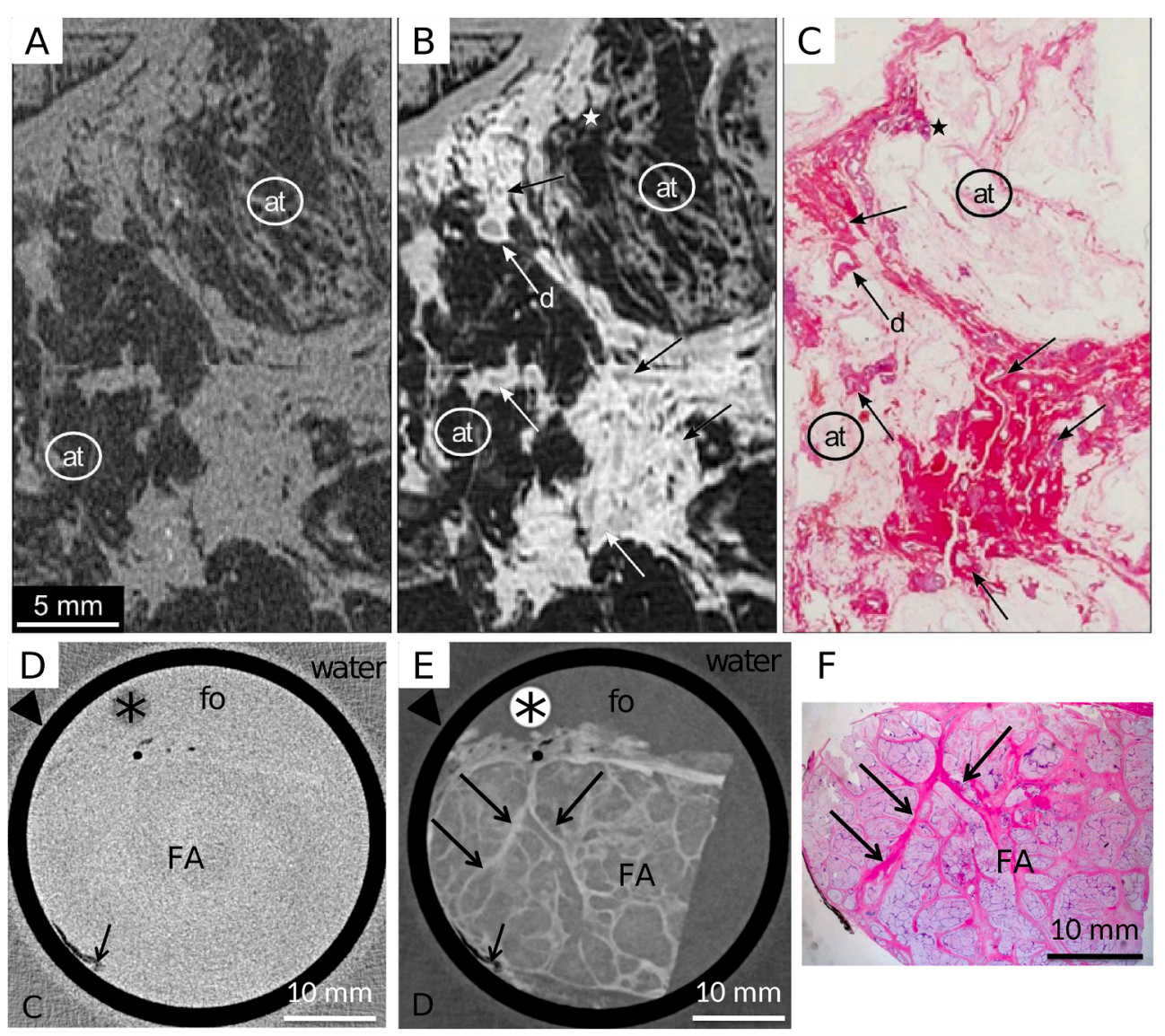

Fig. 6. Grating-based phase-contrast computed tomography examples for ductal carcinoma in situ (DCIS) (A-C) and fibroadenoma (C-E) $[68,69]$. From left to right, the absorption- (A, D), phase-contrast (B, E) and histology $(C, F)$ images of the respective samples are presented. While the attenuation images - except for adipose tissue in (A) have a low contrast for both cases, the phasecontrast image provides detailed information about the internal components of the samples. For DCIS, dilated ducts which are only visible in the phase-contrast image are marked by arrows (B). In contrast to that, a normal duct, with lower signal intensity in the corresponding lumen, is labeled with ' $d$ ' [69]. Asterisk and arrows indicate the DCIS area showing an irregular ductal wall and lumen shape. For the fibroadenoma example (C-E), the absorption-contrast image reveals nearly no information about the internal structure whereas in the phase-contrast image nodular components are very well visible [68]. Bright fibrous tissue strands are marked by long arrows whereas short arrows mark the adhering adipose tissue and arrowheads indicate the plastic container of the sample holder. The histograms confirm the information obtained by the phasecontrast information. Images reprinted from Grandl and Hellerhoff et al. with permission from the authors $[68,69]$.
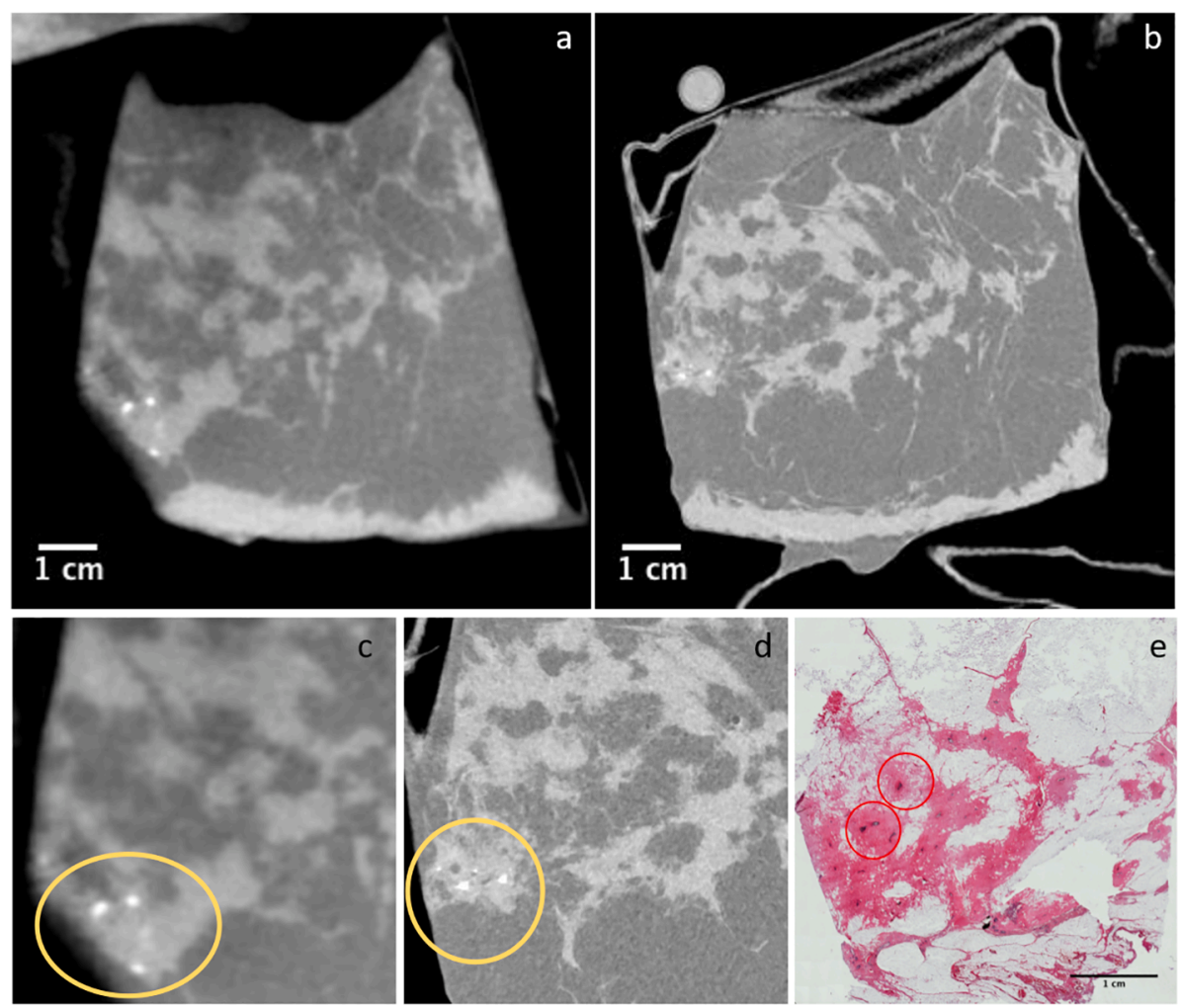

Fig. 7. Comparison of absorption-contrast cone beam BCT (a, c) with phase-contrast BCT (b, d) performed with synchrotron radiation at an energy of $32 \mathrm{keV}$. The results include an invasive ductal carcinoma with DCIS. A better visualization of the lesion morphology, tissue infiltration and the definition of margins is achieved by the synchrotron radiation image. The zoom-in sections (c, d) indicate the microcalcification area from the sample $(a, b)$, respectively. They additionally underline the improved detection and differentiation of the microcalcification cluster including its morphology and distribution. Imaging results are confirmed by histological assessment (e) [79]. Images reprinted from Pacilè et al. with permission from the authors [79]. 
scan of a mastectomy specimen [77] and investigate the effect of the propagation distance on the image quality [76]. In their feasibility study including twelve mastectomy specimens $(3-4 \mathrm{~cm}$ large), Pacilè et al. were the first to evaluate the benefit by comparing propagation-based and conventional BCT [79]. The clinical assessment by two radiologists shows that a higher diagnostic value is achieved, and hence earlier breast cancer detection would be possible with this imaging modality. An example of this study underlining the fundamental progress of propagation-based BCT is shown in Fig. 7 by Pacilè et al., which compares absorption and phase-contrast BCT and shows an improved visualization of lesion morphology, tissue infiltration and margin definition [79]. Based on these promising results, the first full sample scan with a volume of $9 \times 9 \times 3 \mathrm{~cm}^{3}$ has been performed and proves that synchrotronbased phase-contrast and phase-retrieved breast computed tomography in a continuous rotating mode is feasible while meeting requirements such as scan time and volume, spatial resolution and CNR [77]. In their study, they compared different energies ( 32 and $38 \mathrm{keV}$ ), doses ( 5 and $20 \mathrm{mGy}$ ) and reconstruction with and without a phaseretrieval algorithm. They conclude that the combination of phase- and nonphase-retrieved images yields complementary information since the images without phase retrieval obtain a minimum blur whereas the phase-retrieved images have a better visibility for low-contrast breast features. These images were acquired with a propagation distance of 1.6 $\mathrm{m}$. The effect of this distance has additionally been evaluated in another study where three object-to-detector distances were compared (1.6, 3 and $9 \mathrm{~m}$ ) [76]. Based on one sample, Brombal et al. showed that the highest SNR and the best edge enhancement is achieved with the highest propagation distance of nine meters while keeping the spatial resolution constant, which agrees with their theoretical considerations. Another study compares the image quality of phase-contrast BCT with a conventional BCT at approximately the same dose [71]. Therefore, measurements with a breast phantom have been performed with both imaging approaches and finally the signal-to-noise-ratio (SNR), CNR, spatial resolution, noise power spectrum (NPS) and the visibility of small details were compared. The outcome shows a 4-5 times higher spatial resolution and better SNR for the synchrotron radiation based BCT. The smallest fiber structures as well as microcalcifications (clinical limit is $0.2 \mathrm{~mm}$ ) are still detectable in the BCT performed at Elettra. This study has been conducted with a photon energy which is comparable to the mean energy of the commercial BCT system. But in a further simulationbased study, Delogu et al. investigated the optimal energy for this imaging application with the aim to achieve a compromise between applied MGD and the CNR [75]. Different sample thicknesses and breast glandularities have been evaluated yielding an optimal energy of 28 $\mathrm{keV}$. But one must consider that this energy is just optimized for this specific beamline and geometry and can be further influenced by a real detector (due to different energy responses). In addition, the composition of different breast tissues (adipose, glandular, tumor, skin) has been evaluated at the SYRMEP beamline in terms of estimating the linear attenuation coefficient with totally seven samples in an BCT-interesting energy range (26-38 keV) [72]. The results agree with the already existing literature values. Just like it was already done for the gratingbased approach, the propagation-based BCT results have also been successfully correlated with histological images by comparing the visibility of different lesions from interesting areas in both image modalities [74]. Finally, Longo et al. presented the advances for the clinical implementation of this imaging approach [73]. Again, the agreement with histological sections resulted in a more precise classification of the tumors. The scanning time of the experiments was between 5 and $9 \mathrm{~min}$, resulting in an MGD of $5 \mathrm{mGy}$. Three breast specimens have successfully been measured showing a high spatial and contrast resolution and, in addition to the clinically performed mammography approach, a full three-dimensional view of the breast avoiding tissue superposition and enabling a detailed characterization of breast lesions.

Research at the imaging and medical beamline (IMBL) of Australian Synchrotron also focusses on the propagation-based phase-contrast approach for BCT with synchrotron radiation on mastectomy specimens [80-84]. In 2018, the first freshly - not formalin fixated - dissected mastectomy sample was scanned at the Australian Synchrotron with an energy of $32 \mathrm{keV}$ and a MGD of $4 \mathrm{mGy}$ which is comparable to conventional mammography [84]. One study investigates several reconstruction possibilities of a measured sample, including different distances between sample and detector, energies and reconstruction methods. Again an optimal energy of $32 \mathrm{keV}$, the longest distance between sample and detector and the use of the phase retrieval algorithm are evaluated as optimal parameters [80]. All the conducted studies include an image quality assessment of several clinical radiologists or experts by a visual grading system. In every case, an improvement of the image quality and higher accuracy compared to conventional imaging techniques has been evaluated and thus the propagation-based approach outperformed conventional BCT in terms of the radiological score and the image characteristics. One study additionally reveals a higher radiological image quality at a lower MGD [81]. In summary, the recent developments and various studies at Elettra and the Australian synchrotron for propagation-based phase-contrast breast computed tomography achieved a fundamental improvement of diagnostic image content while keeping the dose close to two-view mammography and comparing with a conventionally used BCT.

\section{Closing Remarks}

During the last decade, several fundamental advances in different directions have been achieved for the improvement of early breast cancer detection. This concerns on the one hand the extended investigation of possible applications and the implementation for the clinical use of grating-based phase-contrast mammography. On the other hand, it deals with the advantageous extension of projection-based mammography to three-dimensional breast imaging for absorption- as well as for phase-contrast imaging.

For mammography, numerous studies have been conducted, including various phase-contrast techniques and dose- and non-dose compatible studies with synchrotron radiation and polychromatic Xray sources. All these studies underline the great potential and benefit of phase-contrast imaging for the female breast. Especially the latest developments (cf. Sec. 2.2.) finally strongly push grating-based phase contrast towards a possible prospective clinical implementation since the maximum allowable dose level has been maintained. However, the last step for a final clinical implementation is still pending. Therefore, studies with larger patient cohorts and clinical in vivo studies on a clinical phase-contrast mammography imaging device are necessary to evaluate the full potential of this novel imaging approach in comparison to the standard imaging procedures. In their last publication, Arboleda et al. announced such an in vivo pilot study which would be another milestone for this field of research [54].

Another milestone in the field of X-ray breast imaging is the successful application of computed tomography at the Elettra and Australian synchrotron in combination with propagation-based phase contrast. Their outstanding studies, including the comparison of physical image quality and visual, radiological assessment, proved a better performance compared to conventional absorption based BCT. Although Gureyev et al. finally concluded that this approach can be used in a complementary way in addition to the existing image modalities [82], it is rather unlikely to scan women in vivo at large-scale synchrotron facilities and thus, this approach is still far away from daily clinical application. Nevertheless, these results remain very promising and might be applied to conventional X-ray sources with other phase-contrast approaches.

But up until now, the results obtained so far in terms of grating-based phase-contrast BCT with conventional X-ray sources are mainly designed to compare with clinical histopathology since the applied MGD and exposure times exceed the clinical standards. Benefits such as the improved visibility of breast lesions like fibroadenomas and DCIS compared to conventional tomography have successfully been 
presented. In order to further investigate the quantitative character and the possible added value for pathological diagnostics, more studies with a larger number of samples have to be conducted.

Another application where this approach could be very useful would be the investigation of in vivo grating-based phase-contrast BCT to support imaging diagnostics. Therefore, the previous studies must initially be extended to larger breast specimens instead of small sample sections and then the applied MGD have to be significantly decreased. Just like for phase-contrast mammography, larger patient groups must then be examined for a full evaluation of this approach. It should be mentioned that results of the first dedicated, grating-based phase-contrast breast computed tomography system developed by GratXray AG, founded in 2017, are expected soon [85]. Compared to the successful studies on propagation-based phase-contrast BCT at large-scale synchrotron facilities, this method would greatly benefit from its easy application at conventional X-ray sources widely used within the clinical environment.

\section{Declaration of Competing Interest}

The authors declare that they have no known competing financial interests or personal relationships that could have appeared to influence the work reported in this paper.

\section{Acknowledgements}

We acknowledge financial support through the DFG (Research Training Group GRK 2274) and the support of all authors who provide us with the figures presented in this review article.

\section{References}

[1] WHO. Global Health Observatory (GHO) data: Women and cervical and breast cancer n.d.

[2] WHO. Global Health Observatory (GHO) data: Women and cervical and breast cancer. World Heal Organ 2020. https://www.who.int/gho/women_and_health/ diseases_risk_factors/cancer_text/en/.

[3] WHO. Breast cancer. World Heal Organ 2020. https://www.who.int/cancer/ prevention/diagnosis-screening/breast-cancer/en/.

[4] Hendrick RE, Baker AJ, Helvie AM. Breast cancer deaths averted over 3 decades. Cancer 2019. https://doi.org/10.1002/cncr.31954.

[5] Paci E, Broeders M, Hofvind S, Puliti D, Duffy S. European Breast Cancer Service Screening Outcomes: A First Balance Sheet of the Benefits and Harms. Cancer Epidemiol Biomarkers \& Prev 2014;23:1159-63. https://doi.org/10.1158/10559965.EPI-13-0320.

[6] Taylor R, Morrell S, Estoesta J, Brassil A. Mammography Screening and Breast Cancer Mortality in New South Wales, Australia. Cancer Causes Control 2004;15: 543-50. https://doi.org/10.1023/B:CACO.0000036153.95908.f2.

[7] Paap E, L M Verbeek A, Puliti D, Paci E, Broeders M. Breast cancer screening casecontrol study design: Impact on breast cancer mortality. Ann Oncol 2010;22: 863-9. https://doi.org/10.1093/annonc/mdq44.

[8] E. Paap L.M. Verbeek A, A.M. Botterweck A, van Doorne-Nagtegaal H, Imhof-Tas $\mathrm{M}$, Koning $\mathrm{H}$, et al. Breast cancer screening halves the risk of breast cancer death: A case-referent study Breast 2014;23. 10.1016/j.breast.2014.03.002.

[9] Sankatsing V, van Ravesteyn N, Heijnsdijk E, Looman WNC, van Luijt P, Fracheboud J, et al. The effect of population-based mammography screening in Dutch municipalities on breast cancer mortality: 20 years of follow-up: The effect of population-based mammography screening on breast cancer mortality. Int $\mathrm{J}$ Cancer 2017;141. https://doi.org/10.1002/ijc.30754.

[10] Stout NK, Lee SJ, Schechter CB, Kerlikowske K, Alagoz O, Berry D, et al. Benefits, harms, and costs for breast cancer screening after US implementation of digital mammography. J Natl Cancer Inst 2014. 106:dju092.

[11] Venkatesan A, Chu P, Kerlikowske K, Sickles EA, Smith-Bindman R. Positive predictive value of specific mammographic findings according to reader and patient variables. Radiology 2009;250:648-57.

[12] Mandelson MT, Oestreicher N, Porter PL, Finder DWCA, Taplin SH, White E. Breast Density as a Predictor of Mammographic Detection: Comparison of Interval- and Screen-Detected Cancers. JNCI J Natl Cancer Inst 2000;92:1081-7.

[13] Destounis S, Johnston L, Highnam R, Arieno A, Morgan R, Chan A. Using Volumetric Breast Density to Quantify the Potential Masking Risk of Mammographic Density. AJR Am J Roentgenol 2016;208:1-6. https://doi.org/ 10.2214/AJR.16.16489.

[14] Riedl C, Luft N, Bernhart C, Weber M, Bernathova M, Tea M, et al. Triple-Modality Screening Trial for Familial Breast Cancer Underlines the Importance of Magnetic Resonance Imaging and Questions the Role of Mammography and Ultrasound Regardless of Patient Mutation Status, Age, and Breast Density. J Clin Oncol Off J Am Soc Clin Oncol 2015;33. https://doi.org/10.1200/JCO.2014.56.8626.
[15] Kuhl CK, Schrading S, Bieling HB, Wardelmann E, Leutner CC, Koenig R, et al. MRI for diagnosis of pure ductal carcinoma in situ: a prospective observational study. Lancet 2007;370:485-92. https://doi.org/10.1016/S0140-6736(07)61232-X.

[16] Clevert DA, Jung EM, Jungius K-P, Ertan K, Kubale R. Value of tissue harmonic imaging (THI) and contrast harmonic imaging (CHI) in detection and characterisation of breast tumours. Eur Radiol 2007;17:1-10. https://doi.org/ 10.1007/s00330-006-0325-z.

[17] Berg WA. Combined Screening With Ultrasound and Mammography vs Mammography Alone in Women at Elevated Risk of Breast Cancer. JAMA 2008; 299:2151. https://doi.org/10.1001/jama.299.18.2151.

[18] Autier P, Boniol M, Koechlin A, Pizot C, Boniol M. Effectiveness of and overdiagnosis from mammography screening in the Netherlands: population based study. BMJ 2017;359:j5224. https://doi.org/10.1136/bmj.j5224.

[19] Carmeliet PK, Jain R. Angiogenesis in cancer and other disease. Nature 2000;407: 249-57. https://doi.org/10.1038/35025220.

[20] H. Lusic M. Grinstaff X-Ray Computed Tomography Contrast Agents Chem Rev 2012;113. 10.1021/cr200358s.

[21] Gennaro G, Toledano A, Di Maggio C, Baldan E, Bezzon E, La Grassa M, et al. Digital breast tomosynthesis versus digital mammography: A clinical performance study. Eur Radiol 2010. https://doi.org/10.1007/s00330-009-1699-5.

[22] Skaane P, Bandos AI, Gullien R, Eben EB, Ekseth U, Haakenaasen U, et al. Comparison of Digital Mammography Alone and Digital Mammography Plus Tomosynthesis in a Population-based Screening Program. Radiology 2013;267: 47-56. https://doi.org/10.1148/radiol.12121373.

[23] Ciatto S, Houssami N, Bernardi D, Caumo F, Pellegrini M, Brunelli S, et al. Integration of 3D digital mammography with tomosynthesis for population breastcancer screening (STORM): a prospective comparison study. Lancet Oncol 2013;14: 583-9. https://doi.org/10.1016/S1470-2045(13)70134-7.

[24] Auweter S, Herzen J, Willner M, Grandl S, Scherer K, Bamberg F, et al. X-ray phasecontrast imaging of the breast - Advances towards clinical implementation. Br J Radiol 2014;87:20130606. https://doi.org/10.1259/bjr.20130606.

[25] Arfelli F, Bonvicini V, Bravin A, Cantatore G, Castelli E, Palma LD, et al. Mammography with Synchrotron Radiation: Phase-Detection Techniques. Radiology 2000;215:286-93. https://doi.org/10.1148/radiology.215.1. r00ap10286.

[26] Dreossi D, Abrami A, Arfelli F, Bregant P, Casarin K, Chenda V, et al. The mammography project at the SYRMEP beamline. Eur J Radiol 2008. https://doi. org/10.1016/j.ejrad.2008.04.038.

[27] Castelli E, Tonutti M, Arfelli F, Longo R, Quaia E, Rigon L, et al. Mammography with Synchrotron Radiation: First Clinical Experience with Phase-Detection Technique. Radiology 2011;259:684-94.

[28] Longo R, Tonutti M, Rigon L, Arfelli F, Dreossi D, Quai E, et al. Clinical study in phase- contrast mammography: image-quality analysis. Philos Trans Roy Soc, A 2014;372:20130025.

[29] Olivo A, Castelli E. X-ray phase contrast imaging: From synchrotrons to conventional sources. Riv Nuovo Cim Soc Ital Fis 2014;37:467-508.

[30] Eggl E, Schleede S, Bech M, Achterhold K, Grandl S, Sztrókay A, et al. X-ray phasecontrast tomosynthesis of a human ex vivo breast slice with an inverse Compton $\mathrm{x}$ ray source. EPL (Europhysics Lett 2016;116:68003. https://doi.org/10.1209/ 0295-5075/116/68003.

[31] Eggl E, Grandl S, Sztrókay-Gaul A, Dierolf M, Jud C, Heck L, et al. Dose-compatible grating-based phase-contrast mammography on mastectomy specimens using a compact synchrotron source. Sci Rep 2018;8:15700. https://doi.org/10.1038/ s41598-018-33628-z.

[32] F. Pfeiffer T. Weitkamp O. Bunk C. David Phase retrieval and differential phasecontrast imaging with low-brilliance X-ray sources Nat Phys 2006;2. 10.1038/ nphys265.

[33] Pfeiffer F, Bech M, Bunk O, Kraft P, Eikenberry EF, Brönnimann C, et al. Hard-X-ray dark-field imaging using a grating interferometer. Nat Mater 2008;7:134-7. https://doi.org/10.1038/nmat2096.

[34] Gajdos C, Ian Tartter P, Bleiweiss IJ, Hermann G, de Csepel J, Estabrook A, et al. Mammographic Appearance of Nonpalpable Breast Cancer Reflects Pathologic Characteristics. Ann Surg 2002;235:246-51. https://doi.org/10.1097/00000658200202000-00013.

[35] de Roos MA, van der Vegt B, de Vries J, Wesseling J, de Bock GH. Pathological and Biological Differences Between Screen-Detected and Interval Ductal Carcinoma in situ of the Breast. Ann Surg Oncol 2007;14:2097-104. https://doi.org/10.1245/ s10434-007-9395-7.

[36] Stomper PC, Geradts J, Edge SB, Levine EG. Mammographic Predictors of the Presence and Size of Invasive Carcinomas Associated With Malignant Microcalcification Lesions Without a Mass. Am J Roentgenol 2003;181:1679-84. https://doi.org/10.2214/ajr.181.6.1811679.

[37] Wang Z, Hauser N, Singer G, Trippel M, Kubik-Huch RA, Schneider CW, et al. Noninvasive classification of microcalcifications with phase-contrast X-ray mammography. Nat Commun 2014;5:3797. https://doi.org/10.1038/ ncomms 4797.

[38] Scherer K, Birnbacher L, Willer K, Chabior M, Herzen J, Pfeiffer F. Correspondence: Quantitative evaluation of X-ray dark-field images for microcalcification analysis in mammography. Nat Commun 2016;7:10863. https://doi.org/10.1038/ ncomms10863.

[39] Wang Z, Hauser N, Singer G, Trippel M, Kubik-Huch RA, Schneider CW, et al. Correspondence: Reply to 'Quantitative evaluation of X-ray dark-field images for microcalcification analysis in mammography'. Nat Commun 2016;7:10868. https://doi.org/10.1038/ncomms10868. 
[40] Rauch T, Rieger J, Pelzer G, Horn F, Erber R, Wunderle M, et al. Discrimination analysis of breast calcifications using X-ray dark-field radiography. Med Phys 2020; 47:1813-26. https://doi.org/10.1002/mp.14043.

[41] Scherer K, Braig E, Ehn S, Schock J, Wolf J, Birnbacher L, et al. Improved Diagnostics by Assessing the Micromorphology of Breast Calcifications via X-Ray Dark-Field Radiography. Sci Rep 2016;6:36991. https://doi.org/10.1038/ srep36991.

[42] Forte S, Wang Z, Arboleda C, Lång K, Singer G, Kubik-Huch RA, et al. Can grating interferometry-based mammography discriminate benign from malignant microcalcifications in fresh biopsy samples? Eur J Radiol 2020;129:109077. https://doi.org/10.1016/j.ejrad.2020.109077.

[43] T.H. Jensen M. Bech O. Bunk T. Donath C. David Feidenhans'l R, et al. Directional X-ray dark-field imaging Phys Med Biol 2010;55:3317-23. 10.1088/0031-9155/ 55/12/004.

[44] Scherer K, Birnbacher L, Chabior M, Herzen J, Mayr D, Grandl S, et al. BiDirectional X-Ray Phase-Contrast Mammography. PLoS ONE 2014;9:e93502. https://doi.org/10.1371/journal.pone.0093502.

[45] Grandl S, Scherer K, Sztrókay-Gaul A, Birnbacher L, Willer K, Chabior M, et al. Improved visualization of breast cancer features in multifocal carcinoma using phase-contrast and dark-field mammography: an ex-vivo study. Eur J Radiol 2015; 25:3659-68.

[46] Hauser N, Wang Z, Kubik-Huch RA, Trippel M, Singer G, Hohl MK, et al. A study on mastectomy samples to evaluate breast imaging quality and potential clinical relevance of differential phase contrast mammography. Invest Radiol 2014;49: $131-7$.

[47] Perry N, Broeders M, de Wolf C, Törnberg S, Holland R, von Karsa L. European guidelines for quality assurance in breast cancer screening and diagnosis. Ann Oncol 2008;19:614-22.

[48] Scherer K, Willer K, Gromann L, Birnbacher L, Braig E, Grandl S, et al. Toward Clinically Compatible Phase-Contrast Mammography. PLoS ONE 2015;10: e0130776.

[49] Koehler T, Daerr H, Martens G, Kuhn N, Löscher S, van Stevendaal U, et al. Slitscanning differential x-ray phase-contrast mammography: Proof-of-concept experimental studies. Med Phys 2015;42:1959. https://doi.org/10.1118/ 1.4914420.

[50] Gromann LB, Bequé D, Scherer K, Willer K, Birnbacher L, Willner M, et al. Lowdose, phase-contrast mammography with high signal-to-noise ratio. Biomed Opt Express 2016;7:381. https://doi.org/10.1364/BOE.7.000381.

[51] Schröter TJ, Koch F, Meyer P, Baumann M, Münch D, Kunka D, et al. Large area gratings by $\mathrm{x}$-ray LIGA dynamic exposure for x-ray phase-contrast imaging. J Micro/Nanolithography, MEMS, MOEMS 2017;16:013501. https://doi.org/ 10.1117/1.JMM.16.1.013501.

[52] Schröter TJ, Koch FJ, Meyer P, Kunka D, Meiser J, Willer K, et al. Large field-ofview tiled grating structures for X-ray phase-contrast imaging. Rev Sci Instrum 2017;88:015104. https://doi.org/10.1063/1.4973632.

[53] Arboleda C, Wang Z, Koehler T, Martens G, Van Stevendaal U, Bartels M, et al. Sensitivity-based optimization for the design of a grating interferometer for clinica X-ray phase contrast mammography. Opt Express 2017;25:6349. https://doi.org/ 10.1364/OE.25.006349.

[54] Arboleda C, Wang Z, Jefimovs K, Koehler T, Van Stevendaal U, Kuhn N, et al. Towards clinical grating-interferometry mammography. Eur Radiol 2020;30: 1419-25. https://doi.org/10.1007/s00330-019-06362-x.

[55] Chang CHJ, Sibala LJ, Gallagher HJ, Riley CR, Templeton WA, Beasley VP, et al. Computed tomography of the breast. A preliminary report. Radiology 1977;124: 827-9.

[56] Chang CHJ, L Sibala J, L Fritz S, H Gallagher J, J Dwyer S, Templeton AW. Computed tomographic evaluation of the breast. AJR Am J Roentgenol 1978;131: 459-64. https://doi.org/10.2214/ajr.131.3.459.

[57] H. Joseph Chang MD C, L. Sibala MD J, L. Fritz PhD S, J. Dwyer III PhD S, W. Templeton MD A, Lin MD F, et al. Computed tomography in detection and diagnosis of breast cancer Cancer 1980;46:939-46. 10.1002/1097-0142 (19800815)46:4+<939::AID-CNCR2820461315>3.0.CO;2-L.

[58] M. Boone J, Nelson T, K. Lindfors K, Seibert J. Dedicated Breast CT: Radiation Dose and Image Quality Evaluation Radiology 2002;221:657-67. 10.1148/ radiol.2213010334.

[59] M. Boone J, Kwan A, Yang K, Burkett G, K Lindfors K, Nelson T. Computed Tomography for Imaging the Breast J Mammary Gland Biol Neoplasia 2006;11: 103-11. 10.1007/s10911-006-9017-1.

[60] Lindfors KK, Boone JM, Nelson TR, Yang K, Kwan ALC, Miller DF. Dedicated Breast CT: Initial Clinical Experience. Radiology 2008;246:725-33. https://doi.org/ 10.1148/radiol.2463070410.

[61] D. Prionas N, K Lindfors K, Ray S, Huang S, A Beckett L, Monsky W, et al. Contrastenhanced Dedicated Breast CT: Initial Clinical Experience Radiology 2010;256: 714-23. 10.1148/radiol.10092311.

[62] Ballabriga R, Alozy J, Campbell M, Fröjdh E, Heijne E, Koenig T, et al. Review of hybrid pixel detector readout ASICs for spectroscopic X-ray imaging. J Instrum 2016;11:P01007. https://doi.org/10.1088/1748-0221/11/01/P01007.
[63] Kalender WA, Beister M, Boone JM, Kolditz D, Vollmar S, Weigel MCC. Highresolution spiral CT of the breast at very low dose: concept and feasibility considerations. Eur Radiol 2011;22:1-8.

[64] Kalender WA, Kolditz D, Steiding C, Ruth V, Lück F, Roessler A, et al. Technical feasibility proof for high-resolution low-dose photon-counting CT of the breast. Eur Radiol 2016;27:1081-6.

[65] (AB-CT) 2020.

[66] Berger N, Marcon M, Saltybaeva N, Kalender AW, Alkadhi H, Frauenfelder T, et al. Dedicated Breast Computed Tomography With a Photon-Counting Detector: Initial Results of Clinical In Vivo Imaging. Invest Radiol 2019. https://doi.org/10.1097/ RLI.0000000000000552.

[67] Sarno A, Mettivier G, Russo P. Dedicated breast computed tomography: Basic aspects. Med Phys 2015;42:2786-804. https://doi.org/10.1118/1.4919441.

[68] Grandl S, Willner M, Herzen J, Sztrókay-Gaul A, Mayr D, Auweter S, et al. Visualizing Typical Features of Breast Fibroadenomas Using Phase-Contrast CT: An Ex-Vivo Study. PLoS ONE 2014;9:e97101. https://doi.org/10.1371/journal. pone.0097101.

[69] Hellerhoff K, Birnbacher L, Sztrókay-Gaul A, Grandl S, Auweter S, Willner M, et al. Assessment of intraductal carcinoma in situ (DCIS) using grating-based X-ray phase-contrast CT at conventional X-ray sources: An experimental ex-vivo study. PLoS ONE 2019;14:e0210291. https://doi.org/10.1371/journal.pone.0210291.

[70] Willner M, Viermetz M, Marschner M, Scherer K, Braun C, Fingerle A, et al. Quantitative Three-Dimensional Imaging of Lipid, Protein, and Water Contents via X-Ray Phase-Contrast Tomography. PLoS ONE 2016;11:e0151889. https://doi. org/10.1371/journal.pone.0151889.

[71] Brombal L, Arfelli F, Delogu P, Donato S, Mettivier G, Michielsen K, et al. Image quality comparison between a phase-contrast synchrotron radiation breast CT and a clinical breast CT: a phantom based study. Sci Rep 2019;9:17778. https://doi. org/10.1038/s41598-019-54131-z.

[72] Piai A, Contillo A, Arfelli F, Bonazza D, Brombal L, Assunta Cova M, et al. Quantitative characterization of breast tissues with dedicated CT imaging. Phys Med Biol 2019. https://doi.org/10.1088/1361-6560/ab2c29.

[73] Longo R, Arfelli F, Bonazza D, Bottigli U, Brombal L, Contillo A, et al. Advancements towards the implementation of clinical phase-contrast breast computed tomography at Elettra. J Synchrotron Radiat 2019;26:1343-53. https:// doi.org/10.1107/S1600577519005502.

[74] Longo R, Arfelli F, Donato S, Bonazza D, Brombal L, Contillo A, et al. Lesion visibility in phase-contrast breast CT: comparison with histological images. In: Van Ongeval C, Marshall N, Bosmans H, editors. 15th Int. Work. Breast Imaging, SPIE; 2020, p. 68. https://doi.org/10.1117/12.2564202.

[75] Delogu P, Di Trapani V, Brombal L, Mettivier G, Taibi A, Oliva P. Optimization of the energy for Breast monochromatic absorption X-ray Computed Tomography. Sci Rep 2019;9:13135. https://doi.org/10.1038/s41598-019-49351-2.

[76] L. Brombal S. Donato D. Dreossi F. Arfelli D. Bonazza A. Contillo et al. Phasecontrast breast CT: the effect of propagation distance Phys Med Biol 2018;63: 24NT03. 10.1088/1361-6560/aaf2e1.

[77] Brombal L, Golosio B, Arfelli F, Bonazza D, Contillo A, Delogu P, et al. Monochromatic breast computed tomography with synchrotron radiation: phasecontrast and phase-retrieved image comparison and full-volume reconstruction. J Med Imaging 2018;6:1. https://doi.org/10.1117/1.JMI.6.3.031402.

[78] Brombal L, Golosio B, Arfelli F, Contillo A, Delogu P, Donato S, et al. Monochromatic breast CT: absorption and phase-retrieved images. In: Chen G-H, Lo JY, Gilat Schmidt T, editors. Med. Imaging 2018 Phys. Med. Imaging, SPIE; 2018, p. 71. https://doi.org/10.1117/12.2293088.

[79] Pacilè S, Dullin C, Baran P, Tonutti M, Perske C, Fischer U, et al. Free propagation phase-contrast breast CT provides higher image quality than cone-beam breast-CT at low radiation doses: a feasibility study on human mastectomies. Sci Rep 2019;9: 13762. https://doi.org/10.1038/s41598-019-50075-6.

[80] Tavakoli Taba S, Baran P, Lewis S, Heard R, Pacile S, Nesterets YI, et al. Toward Improving Breast Cancer Imaging: Radiological Assessment of Propagation-Based Phase-Contrast CT Technology. Acad Radiol 2019;26:e79-89. https://doi.org/ 10.1016/j.acra.2018.07.008

[81] Tavakoli Taba S, Baran P, Nesterets YI, Pacile S, Wienbeck S, Dullin C, et al. Comparison of propagation-based CT using synchrotron radiation and conventional cone-beam CT for breast imaging. Eur Radiol 2020. https://doi.org/ 10.1007/s00330-019-06567-0.

[82] Gureyev TE, Nesterets YI, Baran PM, Taba ST, Mayo SC, Thompson D, et al. Propagation-based x-ray phase-contrast tomography of mastectomy samples using synchrotron radiation. Med Phys 2019;46:5478-87. https://doi.org/10.1002/ mp.13842.

[83] Tavakoli Taba S, Arhatari BD, Nesterets YI, Gadomkar Z, Mayo SC, Thompson D, et al. Propagation-Based Phase-Contrast CT of the Breast Demonstrates Higher Quality Than Conventional Absorption-Based CT Even at Lower Radiation Dose. Acad Radiol 2020. https://doi.org/10.1016/j.acra.2020.01.009.

[84] Pacilè S, Baran P, Dullin C, Dimmock M, Lockie D, Missbach-Guntner J, et al. Advantages of breast cancer visualization and characterization using synchrotron radiation phase-contrast tomography. J Synchrotron Radiat 2018;25:1460-6. https://doi.org/10.1107/S1600577518010172.

[85] GratXray. GratXray - Home 2020. https://www.gratxray.com/. 